\title{
A new cross spectral method to locate sound sources in one dimensional space
}

\author{
Akihiro Shima,* Jiaxiang Liu,** Hiroyuki Noto, ${ }^{* * *}$ Masato Abe,* \\ and Ken'iti Kido* \\ * Research Center for Applied Information Sciences, Tohoku University, \\ 2-1-1, Katahira, Sendai, 980 Japan \\ **Seihoku Institute of Technology, \\ Seian, People's Republic of China \\ ***Oki Electric Industrial Co., Ltd., \\ 550-5, Higashi-Asakawa-cho, Hachioji, 193 Japan
}

(Received 21 April 1986)

\begin{abstract}
In this paper we describe a new method of locating sound sources in one dimensional space such as in a tube. Several methods have been proposed up to now to locate sound sources using cross spectral or correlation techniques. It is impossible, however, to locate sound sources by such conventional methods in cases where there is more than one sound source or when there are reflecting points in the tube. Therefore, we propose here a new method of locating sound sources using several sensors. In this method, the position of a sound source is first assumed, and the transfer functions from the assumed sound source to all the sensors are calculated using the sound velocity and the distances between them. After compensating the transfer characteristics, the cross spectra between all the pairs of sensor outputs are averaged. The assumed source point is scanned all over the tube. If the assumed source point coincides with the actual source point, the magnitude of the averaged cross spectrum takes a large value. However, the magnitude is less if the assumed point does not coincide with the actual point. The usefulness of this method is confirmed by both the computer simulation and the experiments using an electric shaker and a stream of water leaking from a hole in pipe.
\end{abstract}

PACS number: 43. 50. Yw, 43. 60. Gk, 43. 60. Qv, 43. 85. Ta

\section{INTRODUCTION}

Several methods have been proposed to locate a sound source. ${ }^{1,2)}$ First, there is the method of estimating time differences between all the pairs of several sensor outputs. The position of a sound source is estimated from the time differences if the sound velocity is known. ${ }^{3)}$ However, this method does not work well in cases where there are several sound sources correlated with each other.

In this paper, we propose a method to locate sound sources in one dimensional space, ${ }^{7-9)}$ such as in a water or gas pipe. Sound due to leakage from a pipe reflects at the end of the pipe or at the bent point of the pipe. The conventional method doesn't work well since such the reflected sound has a correlation with the original leakage sound. On the other hand, the method proposed in this paper works well even in such the cases, though it requires many sensors and much more computation. The effectiveness of this method of locating a leakage source is confirmed by a computer simulation and experiments. In the experiments, a soft vinyl pipe filled with air and a hard vinyl pipe filled with water are used.

\section{PRINCIPLE}

This paper deals with a case where the sounds generated by some sources propagate through a 


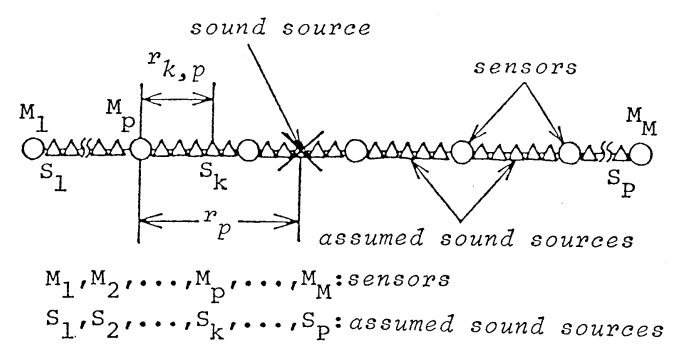

Fig. 1 Relation between the sound source and the sensors.

single line, and a number of sensors are set on the line. Figure 1 shows the relation between the sources and the sensors on the line.

The output signal of every sensor is multiplied by the inverse transfer function from an assumed sound source to a specified sensor. If an actual source is at the assumed source point, the result is the signal of the source with noise due to other sources. The signal of the source will be the same but the noise will be different for different sensors. The cross spectra between the multiplied signals are computed for every pair of sensors providing the condition where the assumed source is between the pair of sensors. The cross spectra are averaged for every assumed source point. The averaged cross spectra will have a significant value if the assumed source point is at an actual source, otherwise the averaged cross spectra will be less than those in the former case.

Taking up the signal radiated by the $n$-th source, the output signal $x_{n, p}(t)$ of the $p$-th sensor is expressed as follows:

$$
x_{n, p}(t)=a_{n}\left(t-r_{n, p} / c\right) \exp \left(-\alpha r_{n, p}\right),
$$

where

$r_{n, p}:$ distance between the $n$-th sound source and the $p$-th sensor

$c$ : sound velocity

$a_{n}(t):$ signal generated by the $n$-th sound source

$\alpha$ : attenuation constant.

$x_{n, p}(t)$ is cut out by an adequate time window $w(t)$, and Fourier transformed as follows:

$$
\begin{aligned}
X_{n, p}(\omega) & =\frac{1}{T} \int_{0}^{T} w(t) x_{n, p}(t) \exp (-j \omega t) d t \\
& \fallingdotseq \exp \left(-\alpha r_{n, p}\right) A_{n}(\omega) \exp \left(-j \omega r_{n, p} / c\right),
\end{aligned}
$$

where

$$
A_{n}(\omega)=\frac{1}{T} \int_{0}^{T} w(t) a_{n}(t) \exp (-j \omega t) d t .
$$

When there are $N$ sound sources, the Fourier transform $X_{p}(\omega)$ of the $p$-th sensor output is expressed as follows:

$$
X_{p}(\omega) \doteqdot \sum_{n=1}^{N} \exp \left(-\alpha r_{n, p}\right) A_{n}(\omega) \exp \left(-j \omega r_{n, p} / c\right) .
$$

First, it is assumed that there is a sound source $S_{k}$ at the point $k$. Then, the inverse transfer function $H_{k, p}(\omega)$ is determined by the distance $r_{k, p}$ between the assumed sound source $S_{k}$ and the $p$-th sensor as follows:

$$
H_{k, p}{ }^{-1}(\omega)=\exp \left(j \omega r_{k, p} / c\right) .
$$

In Eq. (5), we neglect the inverse attenuation factor because of the following reason: If there is no sound source at a specified assumed point, the sensor output contains only the signal from the other sound sources and the signal behaves like a noise. If the inverse attenuation factor is accounted into the computation, the effect of the noise is larger in the case where the distance between the assumed point and the sensor is larger. To decrease the effect, the spatial window, which is just the same as the attenuation factor is used, and the final transfer function is expressed by Eq. (5). A computer simulation is carried out to confirm the effect of the spatial window in Chap. 4.

If there is only one sound source at the point $k$, the source spectrum $A_{k}(\omega)$ of the sound generated by the source $S_{k}$ is computed from the observed spectrum $X_{p}(\omega)$ at the point $p$ using the inverse transfer function $H_{k, p}{ }^{-1}(\omega)$ between the point $k$ to the sensor point $p$ as follows:

$$
A_{k}(\omega)=X_{p}(\omega) H_{k, p}{ }^{-1}(\omega) .
$$

When there are several sound sources, the modified spectrum $Y_{k, p}(\omega)$ is computed as follows:

$$
\begin{aligned}
Y_{k, p}(\omega)= & H_{k, p}{ }^{-1}(\omega) X_{p}(\omega) \\
\doteqdot & \sum_{n=1}^{N} \exp \left(-\alpha r_{n, p}\right) A_{n}(\omega) \\
& \times \exp \left\{-j \omega\left(r_{n, p}-r_{k, p}\right) / c\right\} .
\end{aligned}
$$

The cross spectrum between the modified spectrum $Y_{k, p}(\omega)$ for the $p$-th sensor and $Y_{k, q}(\omega)$ for the $q$-th sensor is computed as follows:

$$
\begin{aligned}
W_{k, p, q}(\omega)= & Y_{k, p}(\omega) Y_{k, q}{ }^{*}(\omega) \\
\fallingdotseq & \sum_{n=1}^{N} \exp \left(-\alpha r_{n, p}\right) A_{n}(\omega) \\
& \times \exp \left\{-j \omega\left(r_{n, p}-r_{k, p}\right) / c\right\} \\
& \times \sum_{n=1}^{N} \exp \left(-\alpha r_{n, q}\right) A_{n}{ }^{*}(\omega)
\end{aligned}
$$




$$
\begin{aligned}
& \times \exp \left\{j \omega\left(r_{n, q}-r_{k, q}\right) / c\right\} \\
= & \sum_{n=1}^{N}\left|A_{n}(\omega)\right|^{2} \exp \left\{-\alpha\left(r_{n, p}+r_{n, q}\right)\right\} \\
& \times \exp \left[j \omega / c\left\{\left(r_{n, q}-r_{k, q}\right)-\left(r_{n, p}-r_{k, p}\right)\right\}\right] \\
& +\sum_{n=1}^{N} \sum_{i=1}^{N} A_{n}(\omega) A_{i}^{*}(\omega) \\
& \times \exp \left\{-\alpha\left(r_{n, p}+r_{i, q}\right)\right\} \\
& \times \exp \left[j \omega / c\left\{\left(r_{i, q}-r_{k, q}\right)-\left(r_{n, p}-r_{k, p}\right)\right\}\right] \\
= & \sum_{n=1}^{N}\left|A_{n}(\omega)\right|^{2} \exp \left\{-\alpha\left(r_{n, p}+r_{n, q}\right)\right\} \\
& \times R_{k}(\omega, n, q, n, p) \\
& +\sum_{n=1}^{N} \sum_{i=1}^{N} A_{n}(\omega) A_{i}^{*}(\omega) \\
& \times \exp \left\{-\alpha\left(r_{n, p}+r_{i, q}\right)\right\} \\
& \times R_{k}(\omega, i, q, n, p),
\end{aligned}
$$

where $A_{n}{ }^{*}(\omega)$ denotes the complex conjugate of $A_{n}(\omega)$,

$$
R_{k}(\omega, i, q, n, p)=\exp \left[j \omega / c\left\{\left(r_{i, q}-r_{k, q}\right)-\left(r_{n, p}-r_{k, p}\right)\right\}\right] .
$$

$W_{k, p, q}(\omega)$ is computed for all the combinations of sensors and averaged as follows:

$$
\begin{aligned}
W_{k}(\omega)= & \frac{1}{K} \sum_{p=1}^{M} \sum_{\substack{q=1 \\
q \neq p}}^{M} W_{k, p, q}(\omega) \\
= & \sum_{n=1}^{N} \frac{\left|A_{n}(\omega)\right|^{2}}{K} \\
& \times \sum_{p=1}^{M} \sum_{\substack{q=1 \\
q \neq p}}^{M} \exp \left\{-\alpha\left(r_{n, p}+r_{n, q}\right)\right\} R_{k}(\omega, n, q, n, p) \\
& +\sum_{n=1}^{N} \sum_{\substack{i=1 \\
i \neq n}}^{N} \frac{A_{n}(\omega) A_{i}^{*}(\omega)}{K} \\
& \times \sum_{p=1}^{M} \sum_{\substack{q=1 \\
q \neq p}}^{M} \exp \left\{-\alpha\left(r_{n, p}+r_{i, q}\right)\right\} R_{k}(\omega, i, q, n, p),
\end{aligned}
$$

where $K$ denotes the number of averaging.

That is, cross spectra $W_{k, p, q}(\omega)$ are averaged $K$ times only in the case where the assumed sound source $S_{k}$ is between the $p$-th sensor and the $q$-th sensor.

The position of the assumed source is scanned along the line to search for the sound sources. The positions of peaks in the averaged cross spectrum represent the locations of sound sources.

Even if the number of the sensors is sufficient, many peaks may be observed at the positions where there is no sound source because of the spatial aliasing. That is, even if the location of the assumed sound source $S_{k}$ is not the same as that of any real sound source, $R_{k}(\omega, n, q, n, p)$ may take the same

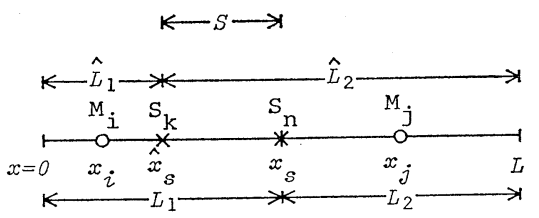

Fig. 2 Relation between the sources and the sensors. $S_{n}$ : sound source, $S_{k}$ : assumed sound source, $M_{i}, M_{j}$ : sensors.

values at $n \neq k$.

The spatial aliasing is discussed in the simple conditions in the following. The sensors are set at equispaces on the tube. There is one sound source, no reflecting sound and no attenuation as shown in Fig. 2. Then, the following equation is obtained from Eq. (9).

$$
w_{k}(\omega)=\frac{W_{k}(\omega)}{\left|A_{n}(\omega)\right|^{2}}=\frac{1}{K} \sum_{p=1}^{M} \sum_{\substack{q=1 \\ q \neq p}}^{M} R_{k},
$$

where

$$
\begin{gathered}
R_{k}=\exp \left[j k\left\{\left(r_{n, q}-r_{k, q}\right)-\left(r_{n, p}-r_{k, p}\right)\right\}\right], \\
k=\frac{\omega}{c} .
\end{gathered}
$$

$M_{1}$ sensors are arranged at left hand side of the assumed sound source $S_{k}$, and $M_{2}$ sensors are at right hand side. The number of averaging $K$ is expressed as follows:

$$
K=2 M_{1} M_{2},
$$

where

$$
M_{2}=M-M_{1} .
$$

Therefore, $w_{k}(\omega)$ is rewritten as follows:

$$
\begin{aligned}
w_{k}(\omega)= & \frac{1}{2 M_{1} M_{2}} \sum_{i=1}^{M_{1}} \sum_{j=1}^{M_{2}}\left(R_{k}+R_{k}^{*}\right) \\
= & \frac{1}{M_{1} M_{2}} \sum_{i=1}^{M_{1}} \sum_{j=1}^{M_{2}} \cos k\left\{\left(r_{n, i}-r_{k, i}\right)\right. \\
& \left.-\left(r_{n, j}-r_{k, j}\right)\right\} .
\end{aligned}
$$

$L$ is the length of the tube, and $x_{s}, \hat{x}_{s}, x_{i}$ and $x_{j}$ are the locations of the real sound source $S_{n}$, the assumed sound source $S_{k}, i$-th sensor and $j$-th sensor, respectively.

If $S_{k}$ is located at the left side of $S_{n}$, and $M_{2}^{\prime}$ the number of sensors which exist between $S_{n}$ and $S_{k}$,

$$
\begin{aligned}
w_{k}(\omega)= & \frac{1}{M_{1} M_{2}} \sum_{i=1}^{M_{1}}\left\{\sum_{j=1}^{M_{2}{ }^{\prime}} \cos 2 k\left(x_{j}-\hat{x}_{s}\right)\right. \\
& \left.+\sum_{j=M_{2^{\prime}}+1}^{M_{2}} \cos 2 k\left(x_{s}-\hat{x}_{s}\right)\right\} .
\end{aligned}
$$


The interval of the sensors is $\Delta x$. Then,

$$
\begin{aligned}
& M_{1} \fallingdotseq \frac{\hat{L}_{1}}{\Delta x} \\
& M_{2} \fallingdotseq \frac{\hat{L}_{2}}{\Delta x},
\end{aligned}
$$

where

$$
\begin{aligned}
& \hat{L}_{1}=\hat{x}_{s} \\
& \hat{L}_{2}=L-\hat{x}_{s} .
\end{aligned}
$$

If the number of sensors is increased, $\Delta x$ approaches zero. Therefore, Eq. (13) is rewritten as follows:

$$
\begin{aligned}
w_{k}(\omega)= & \frac{1}{\hat{L}_{1} \hat{L}_{2}} \int_{0}^{\hat{x}_{s}} d x\left\{\int_{\hat{x}_{s}}^{x_{s}} \cos 2 k\left(x-\hat{x}_{s}\right) d x\right. \\
& \left.+\int_{x_{s}}^{L} \cos 2 k\left(x_{s}-\hat{x}_{s}\right) d x\right\} \\
= & \frac{S}{L_{2}+S} \frac{\sin 2 k S}{2 k S}+\frac{L_{2}}{L_{2}+S} \cos 2 k S,
\end{aligned}
$$

where

$$
\begin{aligned}
L_{2} & =L-x_{s} \\
S & =x_{s}-\hat{x}_{s} .
\end{aligned}
$$

The average of $w_{k}(\omega)$ between frequency $f_{1}$ and $f_{2}$ is expressed as follows:

$$
\begin{aligned}
\left\langle w_{k}\right\rangle= & \frac{S}{L_{2}+S} \frac{S i \epsilon_{2}-S i \epsilon_{1}}{\epsilon_{2}-\epsilon_{1}} \\
& +\frac{L_{2}}{L_{2}+S} \frac{\sin \epsilon_{2}-\sin \epsilon_{1}}{\epsilon_{2}-\epsilon_{1}},
\end{aligned}
$$

where

$$
\begin{aligned}
& \text { Si }=\int_{0}^{\varepsilon} \frac{\sin x}{x} d x \\
& \epsilon_{1}=\frac{4 \pi S}{c} f_{1} \\
& \epsilon_{2}=\frac{4 \pi S}{c} f_{2} .
\end{aligned}
$$

\section{MEASUREMENT OF PROPAGA- TION CHARACTERISTIC}

The exact sound velocity must be known to locate the sound source by the method described in Chap. 2. In this chapter, a method to measure the sound velocity with the attenuation characteristics will be described.

3.1 A Method of Measuring the Sound Velocity Using Cross-correlation Function

Two vibration pick-ups are fixed on a long slender tube. A shaker is fixed at point $\mathbf{S}$ (shown in Fig. 3)

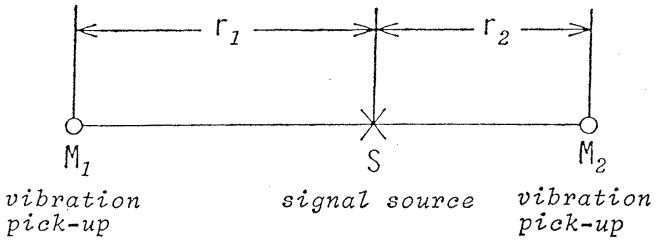

Fig. 3 Relation between the signal source and two vibration pick-ups.

and is driven by white noise. The output signals of the two pick-ups are expressed as follows:

$$
\begin{aligned}
& x(t)=a\left(t-r_{1} / c\right) \exp \left(-\alpha r_{1}\right) \\
& y(t)=a\left(t-r_{2} / c\right) \exp \left(-\alpha r_{2}\right),
\end{aligned}
$$

where

$x(t)$ : output signal of the sensor $M_{1}$

$y(t)$ : output signal of the sensor $M_{2}$

$r_{1}, r_{2}$ : distance between the signal source and each pick-up

$c$ : sound velocity

$a(t):$ signal at the source

$\alpha$ : attenuation constant.

If the sound velocity $c$ changes with the frequency, the frequency band of the output signals $x(t)$ and $y(t)$ should be limited by band pass filters (B.P.F.), so that the sound velocity is constant in the frequency band. Then, the computation of the cross-correlation function between the band-passed signals is carried out as follows:

$$
\begin{aligned}
R_{x y}(\tau)= & \frac{1}{T} \int_{0}^{T} x(t) * w_{\mathrm{B}}(t) y(t-\tau) * w_{\mathrm{B}}(t-\tau) d t \\
= & \exp \left\{-\alpha\left(r_{1}+r_{2}\right)\right\} \\
& \times \frac{1}{T} \int_{0}^{T} a\left(t-r_{1} / c\right) * w_{\mathrm{B}}(t) \\
& \times a\left(t-\tau-r_{2} / c\right) * w_{\mathrm{B}}(t-\tau) d t,
\end{aligned}
$$

where $*$ means the convolution integral, and $w_{\mathrm{B}}(t)$ is the impulse response of the B.P.F.

The cross-correlation function $R_{x y}(\tau)$ takes a maximum value when $\tau$ is equal to $\left(\tau_{1}-\tau_{2}\right)$, where $\tau_{1}=$ $r_{1} / c$ and $\tau_{2}=r_{2} / c$. Therefore, the sound velocity $c$ may be calculated using the following equation:

$$
c=\left(r_{1}-r_{2}\right) / \tau_{\max },
$$

where $\tau_{\max }$ is the time lag at which the cross-correlation function takes the maximum value.

3.2. A Method of Measuring the Sound Velocity Using Coherence Function

The coherence function $\gamma_{x y}(f)$ is defined ${ }^{4)}$ by the 


\section{A. SHIMA et al.: LOCATION OF SOUND SOURCES IN ONE DIMENSIONAL SPACE}

following equation, using the averaged cross spectrum $W_{x y}(f)$ and the averaged power spectra $W_{x x}(f)$ and $W_{y y}(f)$ of the two signals $x(t)$ and $y(t)$.

$$
\gamma_{x y}(f)=W_{x y}(f) / \sqrt{W_{x x}(f) W_{y y}(f)} .
$$

Figure 3 shows the arrangement of the signal source and the two pick-ups. At first, the power spectra $W_{x x}(f)$ and $W_{y y}(f)$ and the cross spectra $W_{x y}(f)$ are computed from the output signals of the two sensors, and the coherence function is computed by Eq. (20). The normalized cross-correlation function $N_{x y}(\tau)$ is obtained by computing the inverse Fourier transform of the coherence function $\gamma_{x y}(f) . \quad N_{x y}(\tau)$ takes a maximum value in the case where $\tau$ is equal to $\left(\tau_{1}-\tau_{2}\right)$ on the time axis. The sound velocity can be calculated using Eq. (19).

$\gamma_{x y}(f)$ can be flat even if the magnitude of the transfer function changes with the frequency, since the coherence function $\gamma_{x y}(f)$ is normalized at every frequency. Therefore, this method may be suitable for measuring the sound velocity when the transfer function changes with the frequency.

\subsection{Experiment on the Measurement of the Sound Velocity}

The experiment was carried out on the measurement of sound velocity using a vinyl chloride pipe. The outer diameter, inner diameter and length of the pipe were $26 \mathrm{~mm}, 20 \mathrm{~mm}$ and $10 \mathrm{~m}$, respectively. Ten equally spaced vibration pick-ups were set on the external surface of the pipe, as shown in Fig. 4. Two types of signal sources were used: one was an electric shaker by which the pipe was vibrated by a white noise, and the other was the stream of water streaming out from a hole in the pipe. The diameter of a

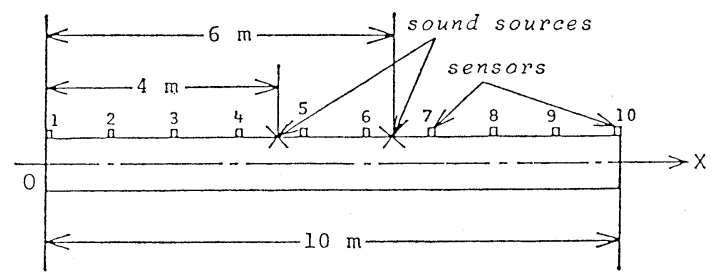

Fig. 4 Relation between the sound sources and the vibration pick-ups for the experiment of measuring the sound velocity.

hole was $3 \mathrm{~mm}$ and the amount of leakage was 4.5 $l / \mathrm{min}$.

The output signals of the 10 vibration pick-ups were amplified and passed through low pass filters with $1.6 \mathrm{kHz}$ cut-off frequency. The low-passed signals were $\mathrm{A} / \mathrm{D}$ converted at a sampling frequency of $3,333 \mathrm{~Hz}$ and stored in a computer. The sound velocity was measured for 4 frequency bands using the cross-correlation function method and the coherence function method described in Sects. 3.1 and 3.2. In the method using a coherence function, the 1,024 points Riesz window $w(n)$ was used, and 10 cross-spectra and 10 power spectra were averaged. The Riesz window is defined as follows.

$$
w(n)=1.0-\left[\frac{n}{\frac{N}{2}}\right]^{2}, \quad 0<|n|<\frac{N}{2} .
$$

To estimate the sound velocity by the cross-spectral technique, the frequency range of the signal should be wide. On the other hand, the sound velocity may have a different value in the different frequency range. Therefore, we estimate the sound velocity for 4

\begin{tabular}{|c|c|c|c|}
\hline \multicolumn{2}{|r|}{ source } & \multirow[t]{2}{*}{ method } & \\
\hline position & $k$ ind & & \\
\hline$s=4.0 \mathrm{~m}$ & shaker & a & $\rightarrow$ \\
\hline $\mathrm{s}=6.0 \mathrm{~m}$ & shaker & a & $\rightarrow$ \\
\hline $\mathrm{s}=4.0 \mathrm{~m}$ & shaker & $b$ & 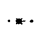 \\
\hline $\mathrm{s}=6.0 \mathrm{~m}$ & shaker & $b$ & + \\
\hline $\mathrm{s}=4.0 \mathrm{~m}$ & leakage of water & a & $\rightarrow$ \\
\hline $\mathrm{s}=6.0 \mathrm{~m}$ & leakage of water & a & $\rightarrow$ \\
\hline $\mathrm{s}=4.0 \mathrm{~m}$ & leakage of water & $b$ & $\cdot x \cdot$ \\
\hline$s=6.0 \mathrm{~m}$ & leakage of water & b & $\cdots$ \\
\hline
\end{tabular}

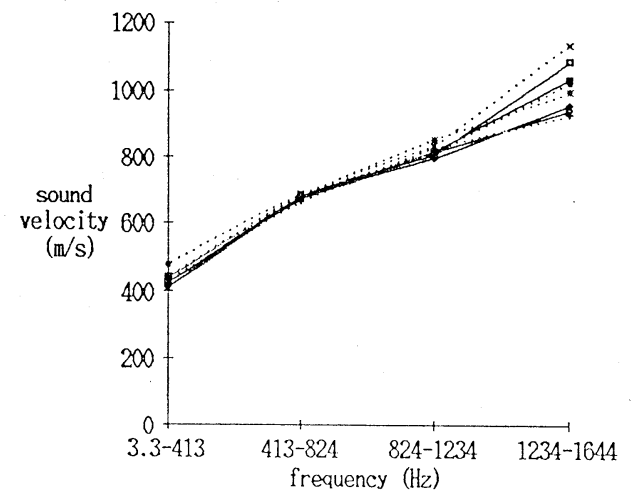

Fig. 5 Results of the measurement of sound velocity. $s$ : distance from the end of pipe (see Fig. 3), $a$ : correlation function method, $b$ : coherence function method. 
frequency bands assuming that the sound velocity is nearly the same in each frequency band. Figure 5 shows the sound velocity estimated both by a correlation function and a coherence function. It is found from this figure that the sound velocity is expressed as follows:

$$
c \fallingdotseq c_{0}+a f,
$$

where

$$
\begin{aligned}
c: & \text { sound velocity } \\
c_{0}: & \text { sound velocity at } f=0 \mathrm{~Hz} \\
a: & \text { constant } \\
f: & \text { frequency. }
\end{aligned}
$$

Similar results are obtained with the correlation function method and the coherence function method.

\subsection{Experiment on the Measurement of Attenuation Characteristic}

The experiment on the measurement of attenuation characteristic was carried out under the same conditions as in Sect. 3.3. Figure 6 shows the smoothed transfer function, which is obtained using the following moving-average function:

$$
w_{n}=\left\{w_{n-2}+w_{n-1}+w_{n}+w_{n+1}+w_{n+2}\right\} / 5 .
$$

The attenuation constant is calculated from the smoothed transfer function. Figure 8 shows the attenuation constant calculated for each frequency component. As shown in Fig. 7, the attenuation

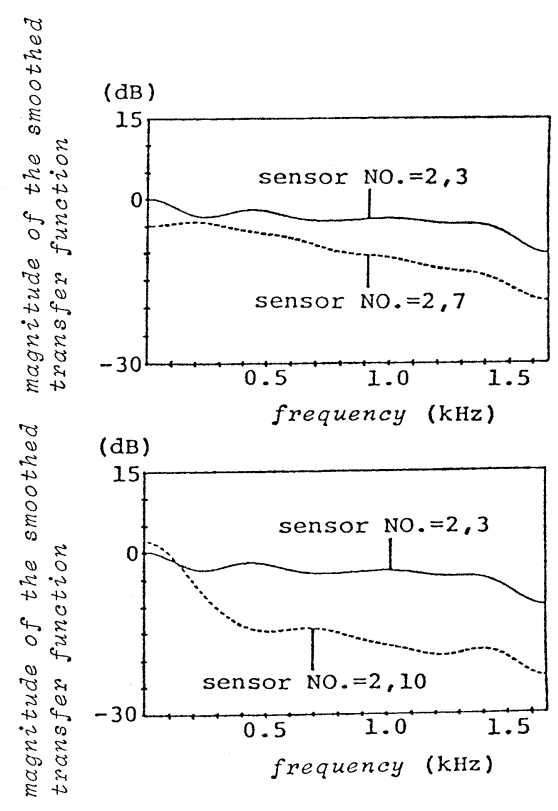

Fig. 6 The smoothed transfer function. characteristics cannot be expressed as constant at all frequency bands. This is because many vibration modes occur in the case of vinyl pipe.

\section{LOCATION OF SOUND SOURCES}

\subsection{Computer Simulation}

The outline of the tube assumed in the computer simulation is shown in Fig. 8. The reflecting points were set at both ends of the tube. The reflecting coefficient was 0.8. A sound source was used to radiate white noise. In each simulation the number of sensors used was increased by a factor of 2 , beginning with 2 sensors and ending with 32 . They were arranged at equal spaces on the external surface of the tube. The sound velocity was $400 \mathrm{~m} / \mathrm{s}$, the
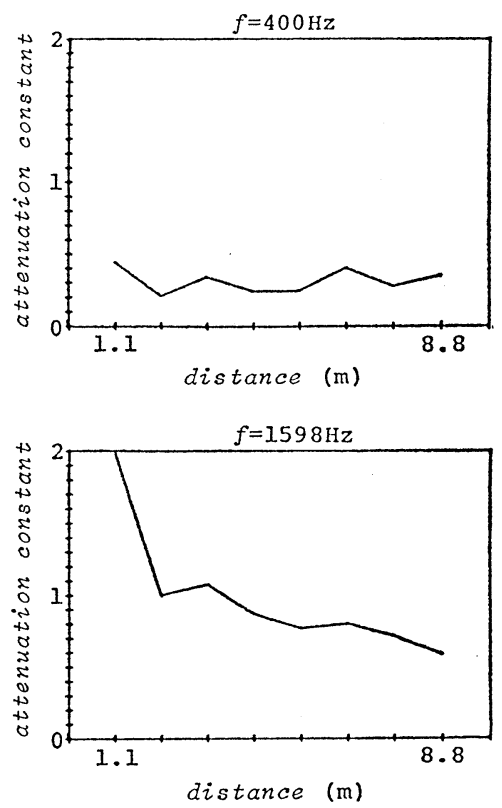

Fig. 7 The attenuation constant.

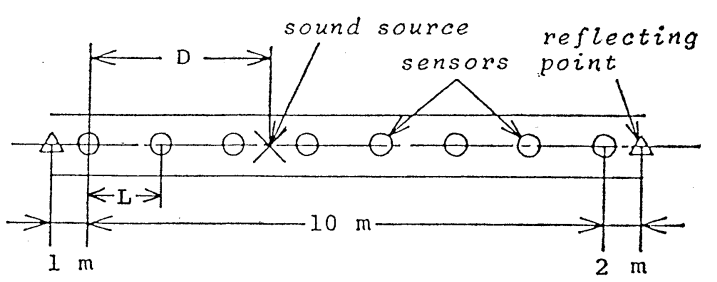

Fig. 8 Relation between the sound source and the sensors for the computer simulations. $D=1,2,3,4,5,6,7,8,9,10(\mathrm{~m}), L=$ $10 /(M-1)(\mathrm{m}), M$ : number of sensors. 


\section{A. SHIMA et al.: LOCATION OF SOUND SOURCES IN ONE DIMENSIONAL SPACE}

attenuation constant $\alpha$ was 0.01 , and the sampling frequency was $2 \mathrm{kHz}$. The sampled data were cut out using a 1,024 points Riesz window for DFT. The number of the assumed sound sources was 201 . In the cross spectral method, the cross spectra were averaged at intervals of $3.9 \mathrm{~Hz}$ from $2 \mathrm{~Hz}$ up to 998 Hz. Figures 9 (a) ( c) show the results obtained by the cross spectral method. Here, the horizontal axis stands for the distance, and the vertical axis the absolute value of the averaged cross spectra which is normalized by the maximum value.

Figure 9 (a) was obtained using 32 sensors in a case where there was a sound source and no reflecting sound. Figure 9 (b) was obtained using 32 sensors in a case where there were both a sound source and reflecting sounds. Figure 9 (c) was obtained using 32 sensors in a case where there were two sound sources and reflecting sound. A clear peak is observed at the location of real sound source even in the case of the presence of the reflecting sounds or in the case of the presence of two sound sources. This clear peak observation makes it possible to estimate the location of sound source.

Figures 9 (d) (f) show the results obtained by the conventional correlation technique using two sensors, ${ }^{5)}$ where the time lag is translated into distance using Eq. (19). A clear peak is observed in Fig. 9 (d) at the location of the actual sound source when there is no reflecting sound. Many large peaks due to the reflected sounds are observed in Figs. 9 (e) and (f) in the positions of no sound sources. In such cases it is difficult to estimate the location of the sound source.

Figure 10 shows the comparison of the results accounting the attenuation by the distance with the results without accounting it. We can obtain better results without accounting the attenuation by the distance.

Figures 11 (a) (c) show the results obtained by the cross spectral method using 32 sensors for each frequency band in a case where there was two sound sources and reflecting sound. We can estimate the location of sound source accurately at high fre-
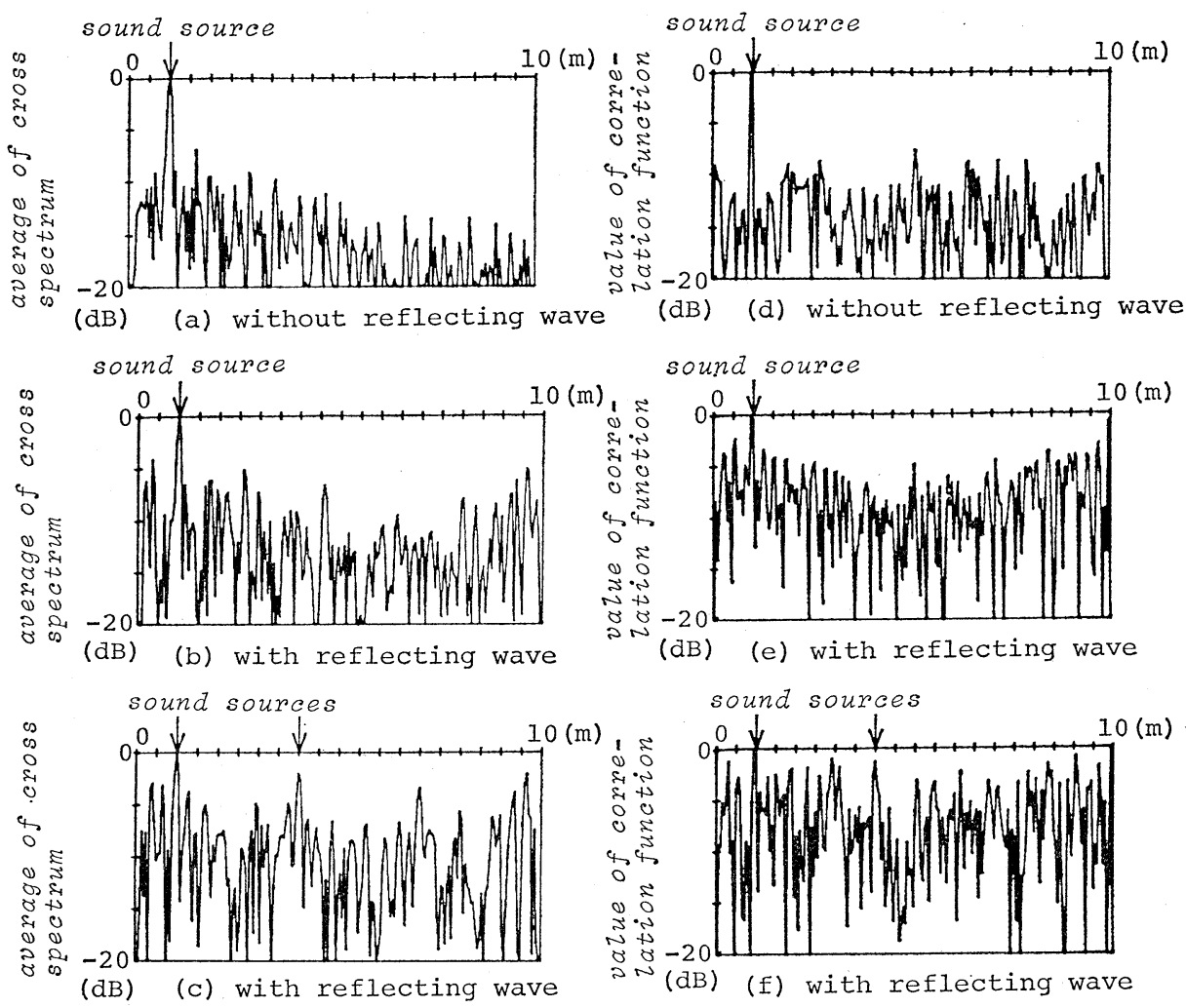

Fig. 9 Results of computer simulation. (a), (b), (c): cross spectral method using 32 sensors, (d), (e), (f): conventional correlation method using 2 sensors. 

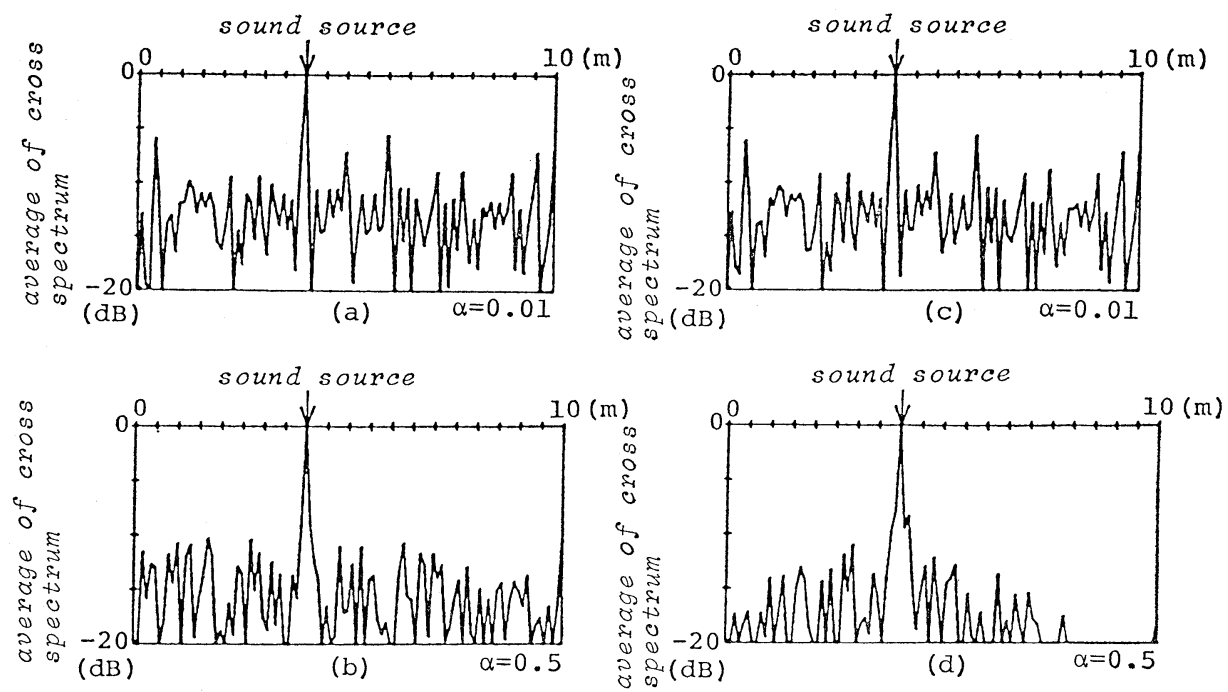

Fig. 10 Comparison of the results accounting the attenuation by the distance with the results without accounting it. (a), (b): accounting the attenuation by the distance, (c), (d): without accounting the attenuation by the distance.
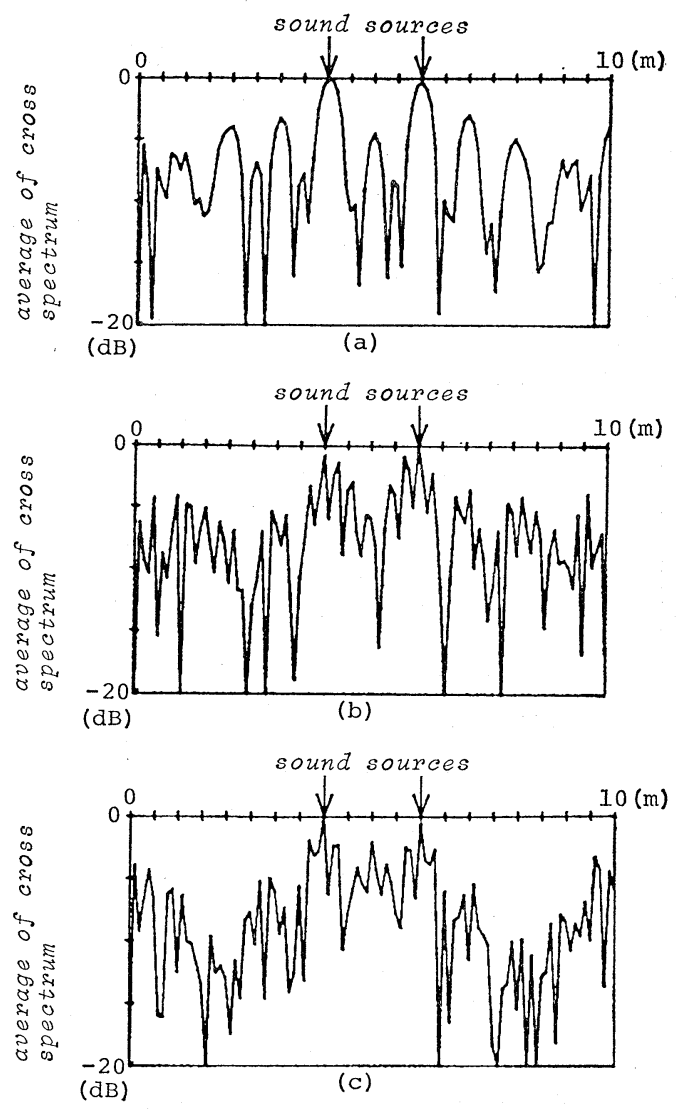

quency band.

To evaluate the easiness of estimation of location of sound source, we introduce an evaluation coefficient, which is the difference between the peak level due to the real sound source and the maximum peak level due to the imaginary source. The larger evaluation coefficient means easier estimation of location of sound source. Figure 12 show the average and the variance of the evaluation coefficient.

Figure 12 (a) shows the change of the evaluation coefficient with the number of sensors where there is a sound source. Figure 12 (b) shows the change of the evaluation coefficient with the number of sensors where there are two sound sources. From these figures it is preferred to use a large number of sensors to find out a real sound source.

Figures 13 (a) $\sim(d)$ show the results of computer simulation using Eqs. (15) and (16). Figures 13 (a) (c) are the results using one frequency component. The frequency is higher, the width of peaks is narrower and the number of peaks increases. Using one frequency component, it is not easy to detect sound

Fig. 11 Results of computer simulation using cross spectral method at each fre䉘 quency band. (a) $3.3 \sim 413 \mathrm{~Hz}$, (b) $413 \sim$ $824 \mathrm{~Hz}$, (c) $824 \sim 1,234 \mathrm{~Hz}$. 


\section{A. SHIMA et al.: LOCATION OF SOUND SOURCES IN ONE DIMENSIONAL SPACE}

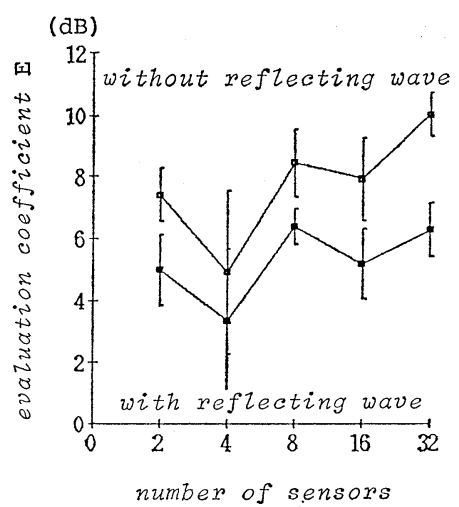

(a)
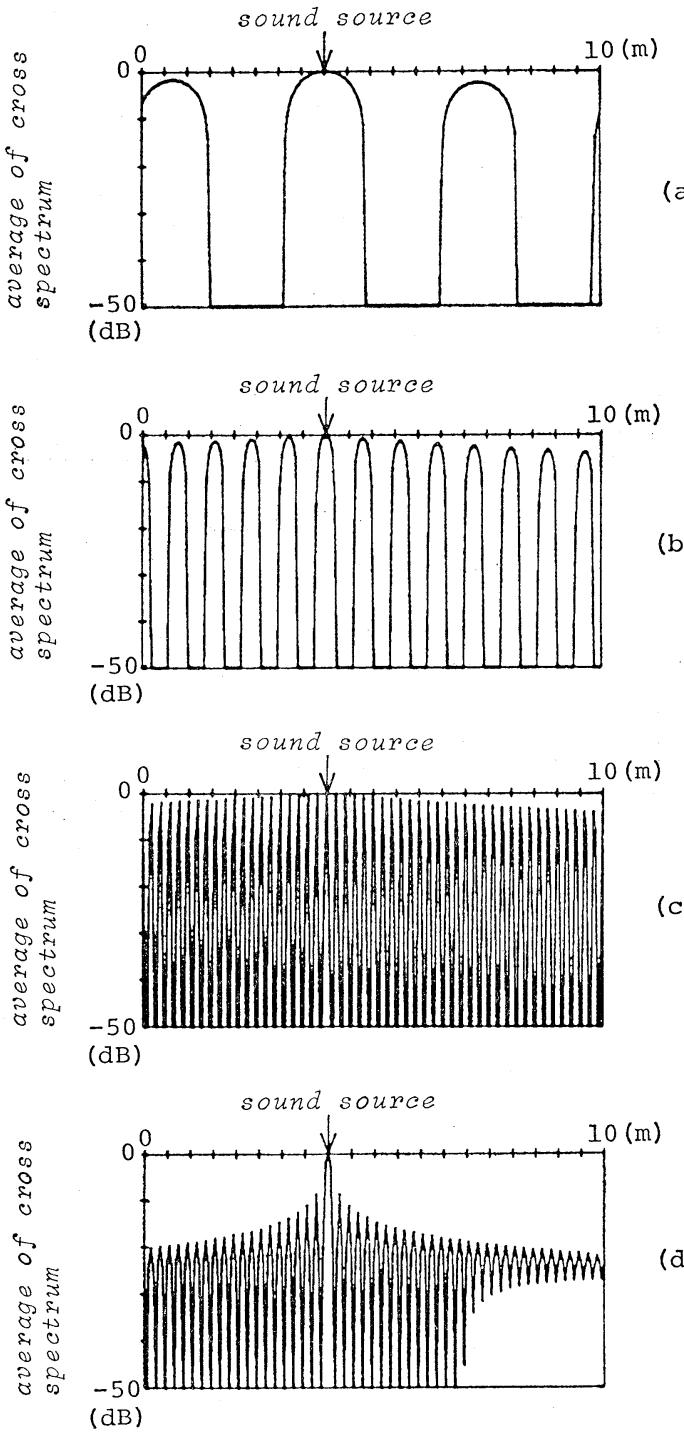

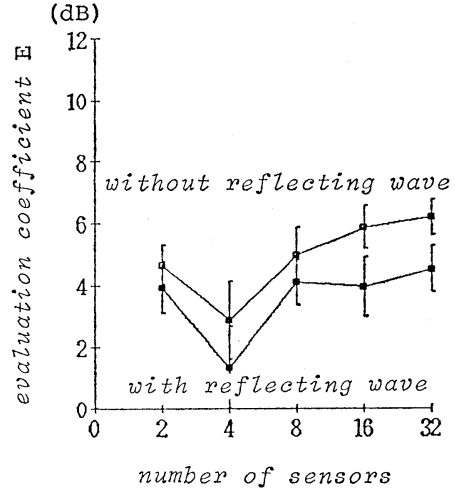

(b)
Fig. 12 Change of evaluation coefficient. (a) one sound source, (b) two sound sources. $E=P_{\mathrm{M}}-P_{\mathrm{n}}(\mathrm{dB})$. $P_{\mathrm{M}}$ : peak level due to the real sound source, $P_{\mathrm{n}}$ : maximum peak level due to the imaginary source. (a)

(b)

(c)

(d)

Fig. 13 Results of computer simulation using continuously distributed sensors. (a) $61 \mathrm{~Hz}$, (b) $248 \mathrm{~Hz}$, (c) $998 \mathrm{~Hz}$, (d) 2 $998 \mathrm{~Hz}$. 


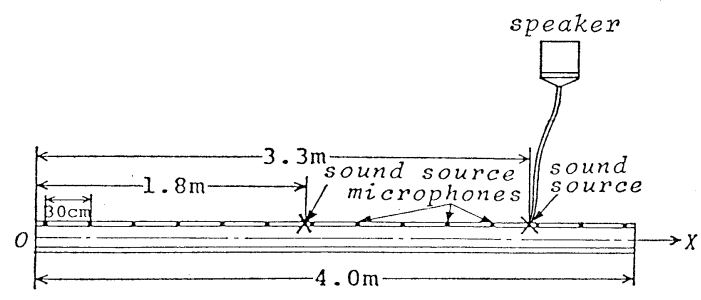

Fig. 14 Relation between the sound source and the microphones for the experiment of an aerial propagation sound.
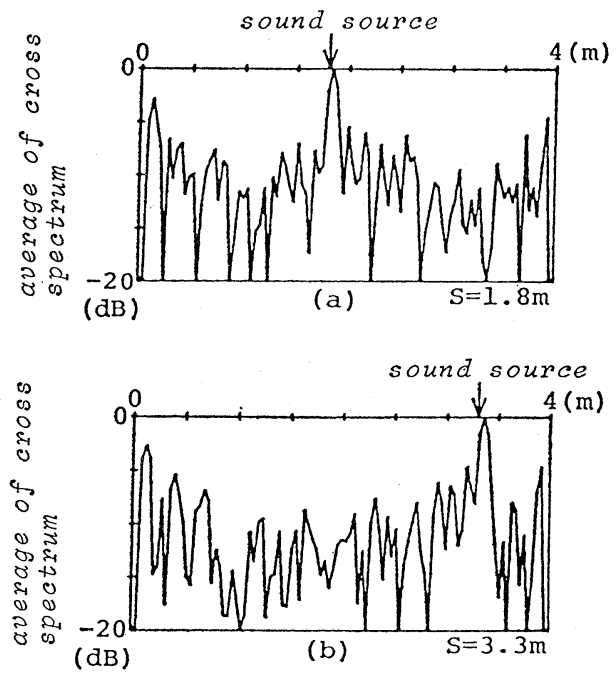

Fig. 15 Results of estimating the location of the sound sources using an aerial propagation.

Figure 15 (a) shows the result in the case where the location of the real sound source was $1.8 \mathrm{~m}$ from the end of pipe. Figure 15 (b) shows the result in the case where the location was $3.3 \mathrm{~m}$ from the end of pipe. In both cases, a peak was observed clearly at the location of the actual sound source.

4.2.2 Electric shaker and the stream of water from a hole

The conditions of the experiment were the same as those of the experiment of measuring the sound velocity described in Sect. 3.3. Figure 16 (a) shows the result in the case where the shaker was located $4 \mathrm{~m}$ from the end of pipe. Figure 16 (b) shows the result in the case where the shaker was set at the point $6 \mathrm{~m}$ apart from the end of pipe. Figure 16 (c) shows the result in the case where the water leaks at a point $4 \mathrm{~m}$
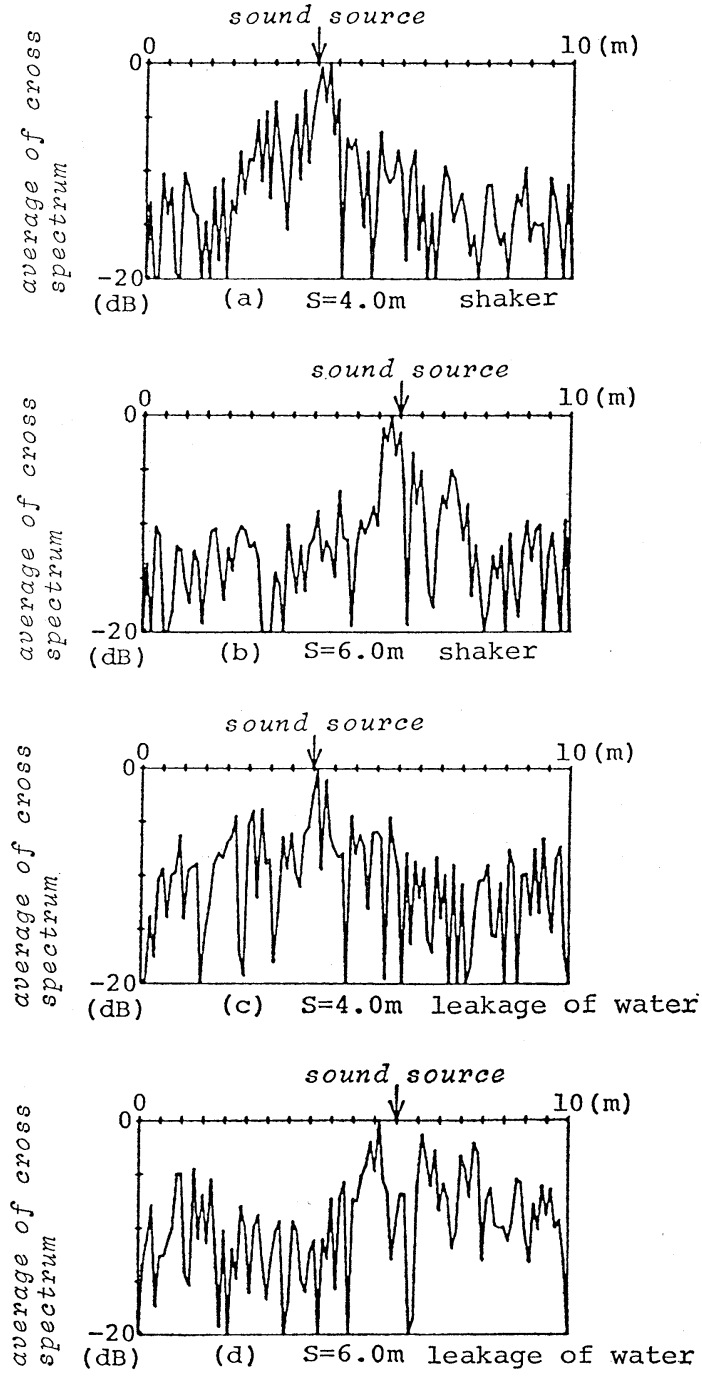

Fig. 16 Results of estimating the location of the sound sources using a shaker and a sound of leakage of water.

from the end of pipe. The diameter of a hole was $3 \mathrm{~mm}$ and the amount of leakage was $4.5 \mathrm{l} / \mathrm{min}$. Figure 16 (d) shows the result in the case where the leak point is at $6 \mathrm{~m}$ from the end of pipe. In every case, a peak is observed very close to the location of the actual sound source.

\section{CONCLUSION}

This paper proposes a new method of locating sound sources in one dimensional space. First, the principle of locating sound sources using a cross 


\section{A. SHIMA et al.: LOCATION OF SOUND SOURCES IN ONE DIMENSIONAL SPACE}

spectral technique was described. Next, a method of measuring the sound velocity using the cross-correlation function and the coherence function was described.

Next, some computer simulations were carried out for locating sound sources. The usefulness of this method was confirmed by these simulations.

Then, some experiments were carried out for measuring the sound velocity by using a vinyl pipe filled with water. The attenuation characteristic was also measured. However, this value was not used in the location of sound sources, since a better estimation is achieved in case of neglecting the attenuation characteristic. Experiments using an electric shaker and a stream of water leaking from a hole in the pipe were carried out, and it is found that the positions of the signal sources could be estimated accurately by the proposed method using the cross spectral technique.

\section{REFERENCES}

1) Y. Arai, "A method of measurement for noise analysis," J. Soc. Instr. Control Eng. 16, 390-418 (1977) (in Japanese).
2) D. H. Youn and N. Ahmed, "Time delay estimation via coherence: An adaptive approach," J. Acoust. Soc. Am. 75, 505-514 (1984).

3) Y. Ishii, "Correlation technique (II)," J. Acoust. Soc. Jpn. (J) 26, 287-291 (1970) (in Japanese).

4) G. C. Carter, "Time delay estimation," Tech. Rep. Nav. Underwater Syst. Cent. 5335, 12-49 (1976).

5) T. Hashimoto, Z. Abe, and T. Kouri, "A new method of water leak detection," J. Soc. Instr. Control Eng. 6, 3-10 (1967) (in Japanese).

6) M. Abe, H. Noto, K. Kido, and Y. Ikegami, "A method for locating sound sources using many sensors," Tech. Rep. IECE EA82-29, 1-6 (1982) (in Japanese).

7) A. Shima, H. Noto, K. Kido, and M. Abe, "A method for locating sound sources using a number of sensors in one dimensional space," Tech. Rep. IECE EA83-30, 17-24 (1983) (in Japanese).

8) A. Shima, J. Liu, H. Noto, M. Abe, and K. Kido, "Estimation of signal source position using cross spectral technique in linear space," Tech. Rep. IECE EA83-79, 15-22 (1984) (in Japanese).

9) J. Liu, A. Shima, M. Abe, and K. Kido, "Estimation of signal source position using correlation technique in linear space," Tech. Rep. IECE EA83-80, 2330 (1984) (in Japanese). 